\title{
化学分析测量数据处理有关概念的相关商榷
}

\author{
岳宣峰 ${ }^{1, *}$ ，㚞金金 1 ，秦丹 ${ }^{1}$, 逯昊文 ${ }^{1}$, 张延妮 ${ }^{2,{ }^{*}}$ \\ 1陕西师范大学化学化工学院, 陕西省生命分析化学重点实验室, 西安 710062 \\ 2 陕西师范大学药用资源与天然药物化学教育部重点实验室, 西安 710062
}

摘要: 按照概念分类和概念定义的一般原则, 对化学分析课程中有关测量数据处理的概念进行了梳理, 包括化学计量学 基本任务、准确度、精密度、误差等, 对现行教材中存在的内容编排、概念用语、概念的定义展开商榷, 并提出相关建 议, 以期为《化学分析》教材建设及学术用语规范提供思路。

关键词: 误差; 准确度; 精密度; 概念分类; 操作性定义

中图分类号: G64; O651

\section{Discussions on the Concepts Related to Metrical Data in Chemical Analysis}

\author{
Xuanfeng Yue ${ }^{1, *}$, Xin Fan ${ }^{1}$, Dan Qin ${ }^{1}$, Haowen Lu ${ }^{1}$, Yanni Zhang ${ }^{2,}{ }^{\star}$ \\ ${ }^{1}$ Key Laboratory of Analytical Chemistry for Life Science of Shaanxi Province, School of Chemistry and Chemical Engineering, \\ Shaanxi Normal University, Xi'an 710062, China. \\ ${ }^{2}$ Key Laboratory of the Ministry of Education for Medicinal Resources and Natural Pharmaceutical Chemistry, \\ Shaanxi Normal University, Xi'an 710062, China.
}

\begin{abstract}
According to the general principles of classification and definition of concepts the concepts in the course of chemical analysis addressing metrical data are sorted out, including the basic tasks of chemometrics, accuracy, precision and error. Discussions are carried out on the arrangement of contents, concept terminology and definition of some concepts in the currently popular textbooks, and relevant suggestions are put forward, in the hope of providing reference for refining the textbooks of 《Chemical Analysis》 and standardizing academic terminology.
\end{abstract}

Key Words: Error; Accuracy; Precision; Concept classification; Operational definition

\section{1 引言}

为了对测量数据进行科学的处理, 现行 《化学分析》教材中普遍介绍了相关化学计量学基础知 识, 并将其应用于对真值的预期。化学测量的基础理论是化学计量学, 它通过数学及统计学方法在 研究对象的 “表观状态” 和 “真实状态” 之间建立联系, 并通过解析测量数据最大限度地提取被研 究对象的有关特征信息。因此, 教材中普遍介绍了误差、绝对误差、相对误差、系统误差、随机误 差、偏差、准确度、精密度等概念, 并对这些概念之间的关联也做了一定解读, 这些内容涉及化学 测量、统计学、数学及语言逻辑等多个学科, 给教材的编写带来了很大的挑战, 现行主要《化学分

收稿: 2021-06-30; 录用: 2021-08-11; 网络发表: 2021-08-20

*通讯作者, Emails: xfyue@snnu.edu.cn (岳宣峰); ynzhang@snnu.edu.cn (张延妮)

基金资助：陕西师范大学研究生教育教学改革研究项目(GERP-20-18) 
析》教材 ${ }^{[1,2]}$ 在涉及以上知识的编排结构上还不甚合理, 一些概念的定义还不够统一, 一些概念的分 类还不够清晰, 还有一些知识存在可能的疏漏甚至错讹, 而这些问题都是分析化学专业共同体进行 理论研究和教育教学时亟待解决的问题。本文基于概念的分类规则和定义规则, 对两本主要《化学 分析》教材中涉及测量数据处理的概念进行了探讨, 指出有关概念的命名、定义、分类、知识编排 等方面存在的问题, 并提出了相应的改进建议。

\section{2 概念的分类和概念的定义}

\section{1 概念分类的属性及其对概念定义的内在要求}

概念分类是一项复杂的工程, 涉及所有学科, 并且需要在一定的哲学观念的指导下进行。相对 于具体学科, 跨学科的概念分类就更为困难, 一方面是由于综合性工程的巨大挑战, 另一方面是众 口难调, 要取得一致的分类标准非常困难; 另外, 哲学思想对这项工程起着纲领性的作用, 而至今 真正取得不同阶层、不同民族、不同国家与文明社会认同的作为科学的哲学还没有出现。因此, 那 种全面统一的概念分类及其体系的实现还有一段相当长的道路要走, 但这并不妨碍在同一学科内部 及交叉学科之间不断对旧概念进行新分类的尝试, 甚至创立新概念。构建基础概念类别应该以语言 学、形式逻辑、辨证逻辑、数学、系统论等学科所涉及的广义对象为基点, 有关概念分类及构建的 注意事项及其与专业术语之间的关联关系见文献 ${ }^{[3]}$ 。概念分类的外延研究要求首先明确概念的定义, 定义的合理描述有助于聚焦概念最关心的关系脉络, 从而提高概念运用的效率。

\section{2 概念的两种定义方法}

在教育研究中，当在不同的层面、从不同的角度、用不同的定义来探讨同一个概念时，往往会 产生很多误解和歧义, 很大一部分原因是由于对所探讨的概念没有明确客观的操作性定义造成的。 所谓概念的操作性定义 (operational definition) 是根据可观察、可测量、可操作的特征来界定概念含义 的方法, 从本质上说, 下操作性定义就是详细描述研究变量的操作程序和测量指标, 美国的物理学 家布里奇的这一观点和思想在1971年被美国的《科学》杂志列为世界五大哲学成就之一, 他认为: 科学上的概念如果要想避免暧昧不清, 最好能以我们 “所采用的测量它的操作方法” 来界定。下操 作性定义的方法、注意事项等见文献 ${ }^{[4]}$ 。所有的实证性研究都必须有测量, 并且所有的测量都必须 把一般的抽象概念变为具体的行为指标, 以便他人重复、验证和交流, 因而在实际研究过程中, 操 作性定义和抽象性定义都是需要的, 应根据研究的性质和目的灵活运用。在给概念下定义的过程中, 抽象性定义可涵盖研究概念所属的基本特征, 解释范围较大, 具有普遍性, 但往往失之笼统, 无法 据此测量和操纵研究的概念; 操作性定义着眼概念的客观可检验性, 对概念的界定清楚明确, 便于 操纵和测量, 但往往只能涉及概念的少数特征, 具有明显的排它性; 当一个概念同时具有两个定义 时, 比较理想的方式是: 先用抽象性定义描述概念的基本特征, 然后再用操作性定义描述概念的操 作程序和测量指标, 前者为后者提供方向并指明意义。

\section{3 《化学分析》教材中测量数据处理有关概念的相关商榷}

按照以上的概念分类和概念定义规则, 对现行两本主要《化学分析》教材中与测量数据处理有 关的概念及相关内容展开商榷, 涉及预期真值的方法、准确度的内涵及其与误差的关系、误差的抽 象性定义、误差的操作性定义及分类、误差的构成、测量的正确性及其判断方法、数值的 “精密度” 及衡量指标、预期真值的区间表达法等, 详细内容如下。

\section{1 预期真值的方法}

现状：教材中有准确度、误差、置信度和置信区间等概念的介绍, 但没有预期真值方法的明确 分类 ${ }^{[1,2]}$ 。

商榷: 以上几个概念之间的关联关系不够明晰(比如: 真值 $\rightarrow$ 预期值 $\rightarrow$ 准确度 $\rightarrow$ 误差, 这些概念 引入教材时未能做好环环相扣), 各个概念适用的领域并未完全明确(比如: 准确度可以用来评价平 
均值法预期真值的效果, 但一般不用于评价区间法预期真值的效果), 涉及的内容在编排上不够紧凑 (将这些概念有机统一起来的起点是: 对真值的预期这个目的, 然后是预期方法的分类, 最后是对这 些预期方法效果的评价指标, 这些内容在现有教材中并未按照这一逻辑编排), 知识结构呼应性不够 强( “准确度” 和 “误差” 涉及到真值, 而 “置信度” 和 “置信区间” 只涉及总体平均值, 其实这些 概念的展开始终都应该和测量工作的根本目的即预期真值要相呼应)。

建议: 为了获得用来表示测量对象某性质的状态的真值 $\mathrm{T}$, 往往先获得研究对象某性质的一组平 行测量结果 $\left(x_{1} 、 x_{2} 、 \ldots, x_{i}\right)$, 然后可以采用该组数值的消除了误差系统项的平均值 $\left(\bar{x}-f_{\mathrm{sys}}\right)$ 或者采用 消除了误差系统项的置信区间 $\left(\bar{x}-f_{\mathrm{sys}} \pm \frac{t \cdot s}{\sqrt{n}}\right)$ 来预期真值, 如果采用前者(其句型一般为: 预期真值 $\mathrm{T}$ 是 $\bar{x}-f_{\mathrm{sys}}$, 或真值的预期值是 $\bar{x}-f_{\mathrm{sys}}$ ), 可用准确度或误差来评价这种预期的效果。真值是恒定值, 而预期值是与测量的重现性和测量的次数有关的变量, 因此, 预期的不确定性是采用平均值法预期 真值时的缺点, 而且这种不确定性也得不到定量的表达, 只能通过不断盲目增加平行测量的次数去 克服; 如果采用置信区间法来预期真值(其句型一般为: 有 $P$ 的把握认为真值属于区间 $\bar{x}-f_{\mathrm{sys}} \pm \frac{t \cdot s}{\sqrt{n}}$ ), 预期的把握程度就是置信度 ( $P$, 一个百分数), 预期真值可能所属的范围就是置信区间, 可用测量值 出现在置信区间内的几率来评价这种预期的效果。显然, 采用区间法来预期真值比采用平均值法来 地更为科学。

\section{2 准确度的内涵及其与误差的关系}

现状: 准确度是指 “测量值与真值的接近程度, 用误差来衡量” " ${ }^{[1]}$ 或 “分析结果与真值的相符程 度, 通常用误差来表示” [2]。误差越小, 准确度越高; 误差越大, 准确度越低 ${ }^{[1,2]}$ 。

商榷: 在描述研究对象某性质的真实状况与其预期状况之间的关系时, 科学家们从两个相对的 角度逐渐发展了两个不同的概念, 分别来描述这两个状态的一致程度及差异程度, 前者对应的是准 确度, 后者对应的是误差。准确度这个概念的抽象性定义可以是 “研究对象某性质的真实状况与其 预期状况的一致程度”, 其操作性定义可以采用文献 ${ }^{[5]}$ 中的描述： accuracy $=\frac{\bar{x}_{i}}{\mathrm{~T}} \times 100 \%$; 准确度的高 低与误差绝对值的大小负相关, 当误差绝对值越小时, 准确度就越高。虽然采用误差或准确度任何 一个概念来定量描述 “研究对象某性质的真实状况与其预期状况之间的关系” 都是可行的, 我们依 然认为没有必要同时采用误差和准确度来进行描述, 这样会增加概念分类的复杂性, 降低概念思维 的效率。准确度不能用误差来 “表示” 或 “衡量”, “表示”一词在语意上提示主体与受体具有相同 的属性, 而 “准确度” 与 “误差” 却是不同的概念; 抽象性的概念或者无形的事物常常需要通过可 测性的性质和有形的具象来 “衡量” , 而 “准确度” 和 “误差”一旦各自都具有了其操作性定义, 两者之间便不存在相互衡量的关系。另外, 在不同教材对 “准确度” 这一概念的描述中, 出现了 “测 量值” 和 “分析结果” 等不一样的措辞, 其实这些措辞都是对同一事物从不同角度的称谓, 但都没 有抓住引入准确度这一概念的初衷, 引入准确度是为了评价平均值预期法的效果, 所以在描述 “准 确度” 时, 建议将 “测量值” 和 “分析结果” 等这些措辞统一改称为 “预期状况或预期值”。离开对 “误差” 的定义, 就无从判断 “误差越小, 准确度越高” 这个说法是否正确, 如果用现有教材 ${ }^{[1,2]}$ 中的 “绝对误差” 或 “相对误差” 代替以上说法中的 “误差”, 逻辑关系显然都不成立, 因为 “误差绝对 值越小/大” 不等于 “误差越小/大”, 这里需要考虑绝对值才行。

建议: 在描述研究对象某性质的真实状况与其预期状况之间的关系时, 科学家们从两个相对的 角度逐渐发展了两个不同的概念, 分别来描述这两个状态的一致程度及差异程度, 前者对应的是准 确度, 后者对应的是误差。准确度是指研究对象某性质的真实状况与其预期状况的接近程度, 一般 采用一个确定的数值表示研究对象某性质的真实状况即真值 $\mathrm{T}$, 而表示预期状态的预期值一般则是 
通过测量获得的, 具有不确定性, 一般采用 $\bar{x}_{i}$, 其数学表达式为 $\bar{x}_{i}=\frac{\sum_{1}^{i} x}{i}, i$ 可以是 $1-+\infty$ 中任意一个 值, 准确度的操作性定义为: accuracy $=\frac{\bar{x}_{i}}{\mathrm{~T}} \times 100 \%$, 准确度的高低与误差绝对值的大小负相关。由 “绝 对误差” 和 “相对误差” 的定义 ${ }^{[1,2]}$ 可知, 误差有正负之分, 误差的绝对值越小, 就意味着测量的准 确度越高, 反之, 误差的绝对值越大, 就意味着测量的准确度就越低。

\section{3 误差的抽象性定义}

现状：对于概念 “误差”，教材中并未提供确切的抽象性定义 ${ }^{[1,2]}$ 。

商榷：相对于 “绝对误差” 及 “相对误差” 这些低一级的概念, “误差” 这个处于分类体系上一 级的概念尚无抽象性定义, 显然有待补充。

建议：误差指的是研究对象某性质的真实状况与其预期状况之间的差别。

\section{4 误差的操作性定义及分类}

现状：现有教材关于两类误差的描述有 “误差有两种表示方法, 绝对误差和相对误差” [1], “误 差可用绝对误差和相对误差来表示” [2]。绝对误差是指 “测定值与真实值的差值, 其表达式为 $E_{\mathrm{a}}=\bar{x}-x \mathrm{~T}$ ” [1]或 “分析结果与真值之差, 其表达式为 $E_{\mathrm{a}}=\bar{x}-\mathrm{T}$ ” ${ }^{[2]}$ 。相对误差是指 “绝对误差相 当于真实值的百分率, 表示式为 $E_{\mathrm{r}}=\frac{E_{\mathrm{a}}}{\mathrm{T}} \times 100 \%$ ” ${ }^{[1]}$ 或 “绝对误差与真值的百分比率, 表示式为 $E_{\mathrm{r}}=\frac{E_{\mathrm{a}}}{x \mathrm{~T}} \times 100 \%{ }^{[2]}$ 。

商榷: “两种表示方法” 暗含 “两种方法的主体是相同的, 不同仅存在于外在的表示形式”, 然 而 “绝对误差” 和 “相对误差” 却着眼于不同的角度, 在内容和形式上都有差别, 因此表述 “误差 有两种表示方法” 有误; 表述 “误差可用绝对误差和相对误差来表示” 既有概念分类的错误又有语 意表达的错误。不同教材中绝对误差的可操作性定义本质相同, 然而表达式中的符号并不统一, 对 应的抽象性描述也不够统一, 将 “分析结果” 和 “测量值” 统一改为 “预期值” 更为合理; 类似的 问题也存在于两本不同教材 ${ }^{[1,2]}$ 对 “相对误差” 的描述中, 另外, 描述的语言在语法及用语上也都有 错误。

建议: 科学上常用一个数值表示研究对象某性质的具体状态, “误差” 定义中的两个状态对应的 两个值分别称为真值 $(\mathrm{T})$ 和预期值 $\left(\bar{x}_{i}\right)$, 对于这两个值的差别, 按照关注角度是绝对还是相对的差异, 分为绝对误差和相对误差, 前者(absolute error) 用 $E^{\mathrm{ab}}$ 表示, 其表达式为 $E^{\mathrm{ab}}=\bar{x}_{i}-\mathrm{T}$, 后者(relative error) 用 $E^{\mathrm{re}}$ 表示, 其表达式为 $E^{\mathrm{re}}=\frac{E^{\mathrm{ab}}}{\mathrm{T}} \times 100 \%$ 。

\section{5 误差的构成}

现状: 按照来源和性质的不同, 误差分为系统误差和随机误差 ${ }^{[1,2]}$ 。系统误差是由某种固定的原 因造成的, 具有重复性、单向性, 又称可测误差。随机误差是由某些难以控制且无法避免的偶然因 素造成的, 大小和正负不定, 无法测量、不可避免且无法校正, 大量平行测量的随机误差服从正态 分布, 且 “用 $\bar{x}_{i}-\mu$ 表示” ${ }^{[1,2]}$ 。

商榷：如果按照教材 ${ }^{[1,2]}$ 中的命名及分类原则, “系统误差” 和 “随机误差” 属于 “误差” 的子级 概念, 那么其应具有母级概念的本质特征 (预期值与真值的差别), 然而 “随机误差” 与真值无关, “系 统误差” 与预期值无关, 显然, 这里存在子级概念外延逾越母级概念的错误。另外, “系统误差” 尚 无可操性定义; “随机误差” 的抽象性定义和可操性定义不是联袂呈现的, 不利于认知的合理建构。

建议: 误差的产生往往是多个因素综合作用的结果, 在平行测量中, 有些因素对误差的贡献呈 现较为固定的特征, 即贡献的大小及正负方向较为固定, 具有重复性、单向性和可测性的特点; 而 其他的因素对误差的贡献呈现出别样的特点, 其特点包括: 贡献的正负会随机变化, 正负贡献出现 
的几率相当; 贡献的大小随机变化, 小贡献出现的几率高, 大贡献出现的几率小, 极大贡献出现的 几率更小。实际上, 每一次测量误差都可能是以上两类因素同时导致的, 但为了更方便地研究这两 类因素对误差贡献的独立特点, 把他们人为分开, 即一类是对误差的贡献呈现固定正负及大小特点 的因素, 另一类是对误差的贡献不呈现固定正负及大小的因素, 我们可以把前者对误差的贡献称为误 差的 “系统项(systematic fraction), 记做 $f_{\text {sys }}$ ”, 把后者对误差的贡献称为误差的 “随机项(random fraction), 记做 $f_{\text {ran }}$, 每一个预期值的误差往往都是由这两部分叠加耦合而构成的, 误差与其 “系统 项” 和 “随机项”之间的关系式为 $E=f_{\mathrm{sys}}+f_{\mathrm{ran}}$ （包括 $E^{\mathrm{ab}}=f_{\mathrm{sys}}^{\mathrm{ab}}+f_{\mathrm{ran}}^{\mathrm{ab}} E^{\mathrm{re}}=f_{\mathrm{sys}}^{\mathrm{re}}+f_{\mathrm{ran}}^{\mathrm{re}}$ ）。

按照绝对误差的定义:

$$
E^{\mathrm{ab}}=\bar{x}_{i}-\mathrm{T}=\frac{\sum_{1}^{i} E_{i}^{\mathrm{ab}}}{i}=\frac{\left(x_{1}-\mathrm{T}\right)+\left(x_{2}-\mathrm{T}\right)+\cdots+\left(x_{i}-\mathrm{T}\right)}{i}=\frac{\sum_{1}^{i} f_{\mathrm{sys} i}^{\mathrm{ab}}}{i}+\frac{\sum_{1}^{i} f_{\mathrm{ran} i}^{\mathrm{ab}}}{i}=\bar{f}_{\mathrm{sys}}^{\mathrm{ab}}+\bar{f}_{\mathrm{ran}}^{\mathrm{ab}}
$$

其中 $f_{\mathrm{sys}}^{\mathrm{ab}}$ 是固定值, 所以 $\bar{f}_{\mathrm{sys}}^{\mathrm{ab}}=f_{\mathrm{sys}}^{\mathrm{ab}}, \bar{f}_{\mathrm{ran}}^{\mathrm{ab}}$ 是多个 “随机项” 的平均值, $\bar{f}_{\mathrm{ran}}^{\mathrm{ab}}$ 的绝对值随着测量次数的 增加而逐渐减小, 当测量次数趋于 $+\infty, \bar{f}_{\mathrm{ran}}^{\mathrm{ab}}$ 趋于 0 , 此时, 误差的表达式为 $E^{\mathrm{ab}}=\bar{x}_{+\infty}-\mathrm{T}=\mu-\mathrm{T}=f_{\mathrm{sys}}^{\mathrm{ab}}$, 其中 $\bar{x}_{+\infty}$ 称为总体平均值, 记作 $\mu$, 可以看出: 当平行测量次数趋于 $+\infty$, 用总体平均值 $\mu$ 去预期真值 时, “绝对误差 $E^{\mathrm{ab}}$ ” 的大小就是误差的 “系统项 $f_{\mathrm{sys}}^{\mathrm{ab}}$ ” 的大小 (换句话说, 所谓绝对误差的 “系统 项” 是指在重复性条件下, 对同一研究对象某性质进行无限多次测量所获的 $\mu$ 与真值 $\mathrm{T}$ 之差), 所谓测 量的绝对误差的 “随机项” 等于研究对象某性质的单个或多个测量值的平均值 $\left(\bar{x}_{i}\right.$ 此时也是对真值 $\mathrm{T}$ 的预期值)与其总体平均值(即 $\mu$ )之差, 即 $\bar{x}_{i}-\mu, i$ 可以是 $1-+\infty$ 中任意一个值。

\section{6 测量的正确性及其判断方法}

现状：无测量的正确性这一概念 ${ }^{[1,2]}$ 。

商榷: 众所周知, 测量的重现性高是其准确度高的必要而不充分条件, 那么是测量的哪个环节 的缺失导致了这个 “不充分” 呢? 是正确性!

建议: 所谓测量的 “正确性” 指的是在某具体测量条件下, 研究对象某性质无限多个测量结果的 平均值(即 $\mu$ )与其真实状态(即真值 $\mathrm{T}$ )的一致性。 “绝对误差” 的 “系统项 $f_{\mathrm{sys}}^{\mathrm{ab}}$ ” 的绝对值的大小反映了 测量方向正确性的高低, 该绝对值越小, 测量的正确性就越高, 反之正确性就越低; 当 “系统项 $f_{\mathrm{sys}}^{\mathrm{ab}}$ ” 为 0 时, 测量的正确性最高, 此时总体平均值 $\mu$ 等于真值 $\mathrm{T}$; “绝对误差” 的 “系统项 $f_{\mathrm{sys}}^{\mathrm{ab}}$ ” 无法通过增 加测量次数的措施而减小或者消除, 其反映了测量过程中某些固定的、系统性的缺陷。

\section{7 数值的 “精密度” 及衡量指标}

现状：精密度表示 “几次平行测定结果之间相互接近的程度” [1]或 “数次测量值相互接近的程 度” ${ }^{[2]}$, “用偏差来衡量” ${ }^{[1]}$ 或 “用偏差来表示” ${ }^{[2]}$; 偏差越小, 精密度越好 ${ }^{[1,2]}$ 。偏差 ${ }^{[1]}$ (绝对偏 差 $\left.{ }^{[2]}\right) d_{i}$ 表示测量值 $\left(x_{i}\right)$ 与平均值 $\left(\bar{x}_{i}\right)$ 的差值。 $\bar{d}_{i}$ 称为单次测量结果的平均偏差, 代表一组测量值中 任何一个数据的偏差, 它最能表示一组数据间的重现性, 常被用来表示分析结果的精密度 ${ }^{[1]}$ 。偏差 ${ }^{[1]}$ $\left(\right.$ 精密度 ${ }^{[2]}$ )也可以用全距表示。在分析化学中, 有时用重现性和再现性表示不同情况下分析结果的精 密度 ${ }^{[1,2]}$ 。

商榷: 在汉语里一旦一个词汇的核心词是 “度”, 在语意上往往意味着需要用具体数字来体现量 的大小, 而 “精密度” 这个概念本身并无操作性定义, 即无可测的量, 它只能与定性描述用语如 “高” 或 “低” 等相关联, 因此, 在抽象表示几个数值之间的 “亲密” 状态时, 也许采用 “精密性” 比 “精 密度” 的名称更为贴切。但精密性可以用其他具有操作性定义的指标来衡量, 比如标准偏差, 精密 性与其衡量指标常被混淆。近年来, 因为混淆精密性的内涵、衡量指标及其意义而在研究生毕业论 文中屡次出现 “方法的精密度小于 $5 \%$ ” (正确的表达应该是 “测量结果的相对标准偏差小于 $5 \%$, 所 以测量结果的精密性较高, 说明方法的重现性较好” 这样的表达, 由此也可见这部分内容急需规范 的紧迫性。“精密度表示几次平行测定结果之间相互接近的程度, 用偏差来衡量, 偏差越小, 精密 
度越好” [1]中的用词 “表示” 应该改成 “是指”，用 “偏差” 来衡量也显然不合适， “偏差” 这个概念 在各种教材中还没有获得统一的操作性定义(即使按照教材 ${ }^{[1]}$ 中的操作性定义 $d_{i}=x_{i}-\bar{x}_{i}, d_{i}$ 也有正负 之分, 因此不具有 “偏差越小, 精密度越好” 的属性), 如果将上句中 “偏差” 改成 “平均偏差及相 对平均偏差”, 说法是成立的。 $\bar{d}_{i}$ 不能 “代表一组测量值中任何一个数据的偏差”, 而是 “体现了一 组测量值中单个测量值的一般偏差”, $\bar{d}_{i}$ 不是 “常被用来表示分析结果的精密度, 最能表示一组数据 间的重现性” “1], 而是 “常被用来衡量一组数值的精密性, 如果这些数值是测量值, 则可反映相关测 量工具、测量方法或测量过程的重现性”。“精密性”一词是统计学上评价数值之间接近程度的术语, 它和数值的来源或用途无关, 而 “重现性(重复性和再现性)” 是评价数据获得工具、方法或过程的重 现程度的术语, 精密性和重现性既有联系, 又有区别, 一组测量值的精密性的高低可以反映其获得 过程相关环节重现性的好坏, 因此 “用重现性表示分析结果的精密度”[1]的说法错误满满。“偏差 ${ }^{[1]}$ (精密度 ${ }^{[2]}$ )也可以用全距表示” 这里既有混淆 “偏差” 与 “精密性” 这两个概念的错误, 也有用语的 不当, 正确的关系和表达应该是 “精密性也可以用全距来衡量”。 $d_{i}$ 在不同的教材中竟然有 “偏差” 和 “绝对偏差” 两个不同的名称, 应该统一起来。

建议: 所谓精密性是指多个数值(在测量学中, 常涉及平行测量的测量结果)之间的绝对或相对 接近程度, 它的高低反映了数据获得过程相关环节的重现性的好坏, 这些环节一般包括测量工具、 测量方法、测量过程等, 衡量精密性的指标有全距、平均偏差及相对平均偏差、标准偏差及相对标 准偏差等。对研究对象某性质进行多次平行测量之后, 获得一系列测量结果 $\left(x_{1} 、 x_{2} 、 \cdots 、 x_{i}\right)$, 这组 数值的平均值为 $\bar{x}_{i}$, 其中每个数值与 $\bar{x}_{i}$ 的差值称为该数值的 “绝对偏差”, 记作 $d_{i}$, 其数学表达式为 $d_{i}=x_{i}-\bar{x}_{i}$, 而这组数值的平均偏差为所有数值绝对偏差的绝对值的算术平均值, 记作 $\bar{d}_{i}$, 其数学表 达式为 $\bar{d}_{i}=\frac{\sum_{1}^{i}\left|d_{i}\right|}{i}$, 它体现了一组数值中单个数值的一般偏差; 而该组数值的相对平均偏差为其平 均偏差占平均值的百分数, 记作 $\mathrm{R} \bar{d}_{i}$, 其数学表达式为 $\mathrm{R} \bar{d}_{i}=\frac{\bar{d}_{i}}{\bar{x}_{i}} 。 \bar{d}_{i}$ 越小, 表示各个数值之间的绝对 差别越小, 各个数值越绝对接近; $\mathrm{R} \bar{d}_{i}$ 越小, 表示各个数值之间的相对差别越小, 各个数值越相对接 近。标准偏差和相对标准偏差, 分别记作 $s$ 和RSD, 作为衡量多个数值之间接近程度的另外一对相关 联的量, 其作用和意义分别与 $\bar{d}_{i}$ 和 $\mathrm{R} \bar{d}_{i}$ 相同, 不同在于 $s$ 和 RSD的数学表达式中引入了 $d_{i}$ 的平方, 从而 增加了 $d_{i}$ 的权重, 使他们拥有了对数值间细微差别更强的分辨能力, 但其计算公式也变地更为复杂, $s$ 和RSD的数学表达式分别为 $s=\sqrt{\frac{\sum d_{i}^{2}}{n-1}}$ 和 $\mathrm{RSD}=\sqrt{\frac{\sum d_{i}^{2}}{n-1}} / \bar{x}_{i}$ 。

\section{8 预期真值的区间表达法}

现状：教材 ${ }^{[1,2]}$ 中目前并无真值的预期区间的表达式, 但都提供了总体平均值 $\mu$ 的置信区间, 而总 体平均值 $\mu$ 所处区间一般由一组平行测量结果而获得, 其表达式为: $\mu=\bar{x}_{i} \pm \frac{t \cdot s}{\sqrt{n}}$ (只知道样本标准偏 差 $s$ 时), 或 $\mu=\bar{x}_{i} \pm \frac{u \cdot \sigma}{\sqrt{n}}$ (已知总体标准偏差 $\sigma$ 时) ${ }^{[1,2]}$ 。

商榷: 测量的目的是为了预期真值, 预期真值时的误差等于系统项 $f_{\mathrm{sys}}$ 加上随机项 $f_{\mathrm{ran}}$, 系统项是 固定值, 随机项出现的规律符合正态分布或者 $t$ 分布, 可以在计算总体平均值 $\mu$ 的基础上再考虑误差的 系统项, 就可以直接预期真值 T所属的区间。另外, 目前教材中给出的总体平均值 $\mu$ 的置信区间只是 各种可能性中的一种, 它是在保证优先考虑数值精密性最好的前提下的置信区间, 有关这一点我们 会另撰文阐述。

建议: 由 $E^{\mathrm{ab}}=\bar{x}_{+\infty}-\mathrm{T}=\mu-\mathrm{T}=f_{\mathrm{sys}}^{\mathrm{ab}}$ 可知 $\mathrm{T}=\mu-f_{\mathrm{sys}}^{\mathrm{ab}}$, 通过 $\mu$ 的置信区间可以进一步获得在以下情 
况下真值的预期区间: $\mathrm{T}=\bar{x}_{i}-f_{\mathrm{sys}}^{\mathrm{ab}} \pm \frac{u \cdot \sigma}{\sqrt{n}}$ (已知某测量值样本的平均值 $\bar{x}_{i}$, 且已知测量值总体的标准 偏差 $\sigma$ ), $\mathrm{T}=x_{i}-f_{\mathrm{sys}}^{\mathrm{ab}} \pm u \cdot \sigma$ (已知测量值总体的标准偏差 $\sigma$, 同时知道任意单个测量值 $x_{i}$ ), $\mathrm{T}=\bar{x}_{i}-f_{\mathrm{sys}}^{\mathrm{ab}} \pm \frac{t \cdot s}{\sqrt{n}}$ (已知某测量值样本的平均值 $\bar{x}_{i}$, 且已知该样本的标准偏差 $s$ ), $\mathrm{T}=x_{i}-f_{\mathrm{sys}}^{\mathrm{ab}} \pm t \cdot s$ (已 知某测量值样本的标准偏差 $s$, 同时知道该样本的某单个测量值 $x_{i}$ ), $\mathrm{T}=x_{i} \pm \infty$ (只知单个测量值 $x_{i}$, 既不知道测量值总体的标准偏差 $\sigma$, 也不知道任何测量值样本的标准偏差 $s$ )。

\section{参 考 文 献}

[1] 武汉大学. 分析化学(上册). 第6版. 北京: 高等教育出版社, 2019.

[2] 华中师范大学, 等. 分析化学(上册). 第4版. 北京: 高等教育出版社, 2018.

[3] 董红晶. 中国科技术语, 2018, 20 (1), 17.

[4] 朱建. 社会科学战线, 1992, No. 1, 49.

[5] Christian, G. D.; Dasgupta, P. K.; Schug, K. A. Analytical Chemistry, 7th ed.; Wiley: New York, USA, 2014; p. 238. 